OPEN ACCESS

UWS Academic Portal

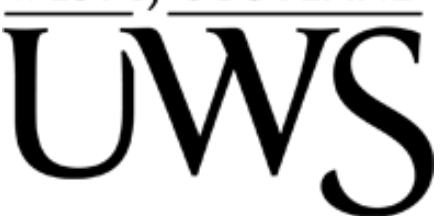

\title{
Naïve Bayesian network for automated, fashion personal stylist
}

Strain, N.; Olszewska, J.I.

Published in:

Proceedings of the International Conference on Agents and Artificial Intelligence

DOI:

$10.5220 / 0009123808140821$

Published: 22/02/2020

Document Version

Peer reviewed version

Link to publication on the UWS Academic Portal

Citation for published version (APA):

Strain, N., \& Olszewska, J. I. (2020). Naïve Bayesian network for automated, fashion personal stylist. In A. Rocha, L. Steels, \& J. van den Herik (Eds.), Proceedings of the International Conference on Agents and Artificial Intelligence (Vol. 2, pp. 814-821). (ICAART). SciTePress. https://doi.org/10.5220/0009123808140821

\section{General rights}

Copyright and moral rights for the publications made accessible in the UWS Academic Portal are retained by the authors and/or other copyright owners and it is a condition of accessing publications that users recognise and abide by the legal requirements associated with these rights.

Take down policy

If you believe that this document breaches copyright please contact pure@uws.ac.uk providing details, and we will remove access to the work immediately and investigate your claim. 


\title{
NAIVE BAYESIAN NETWORK FOR AUTOMATED, FASHION PERSONAL STYLIST
}

\author{
N. Strain and J.I. Olszewska \\ School of Computing and Engineering, University of West Scotland, UK \\ joanna.olszewska@ieee.org
}

\begin{abstract}
Keywords: Intelligent Systems, Agent-Oriented Software Engineering, Expert System, Machine Learning, Bayesian Networks, Knowledge-Based System, Web Intelligence, Data Mining, Industrial Applications of AI.

Abstract: $\quad$ Fashion is an area that people experience every day. Fashion can be seen as homogenizing, since encouraging everyone to dress in a certain way that is influenced, e.g. by celebrities and social media. However, nowadays, fashion is also a search for individuality and personal expression. Hence, this work is about the development of an intelligent web application to help people by providing them with clothing suggestions based on their previous garment selections at the registration stage and after determining each user's individual style thanks to machine learning techniques such as Naive Bayesian Networks. The resulting intelligent system has been thoroughly tested on real-world datasets as well as successfully released to end-users.
\end{abstract}

\section{INTRODUCTION}

Fashion in clothing is a major economic force relying on the design, tailoring, and dissemination of clothes as well as their collections' images, while impacting the social and cultural life and thriving on novelty and change.

Fashion is thought to have started during the Renaissance (Arnold, 2009), with the first fashion magazines appearing in the later 17 th century and increasing fashion visibility and desirability. In the 18th century, a shift occurred from annual changes in textile designs and fashion styles to seasonal changes. By the end of the 19th century, fashion's growth as a driving force within the clothing industry brought stylish clothes to a wider cross-section of people. During the 20th century, 'fast fashion' came to supersede the industry's previous seasonal timetable with regular supplies of new garments sent out to high-street retailers, while fashion consumers have increasingly sought to individualize their look by customizing apparels and mixing designer, high street, and vintage clothes. This has enabled people to act as designers themselves, if not of individual garments, then of the look and image they wish to convey (Arnold, 2009).

The beginning of 21 st century has been marked by the development of the world-wide e-commerce fashion industry, which is currently worth $\$ 545$ billion and is expected to grow to $\$ 712.9$ billion by 2022 (Started, 2019). Online fashion shopping represents
$67 \%$ of UK online purchases, and this figure continues to rise (Started, 2019). For years, leading brands such as Chanel were reluctant to have an online presence, but in order to meet demands of the current digital world, they had to adapt. Indeed, good customer service is no longer what fashion brands need to stay competitive, since the millions of online shoppers look for a fluid, interactive shopping experience (Cecilio, 2015). On the other hand, nowadays, fashion is not solely depending on the latest issue of Vogue to keep people up to date with the latest trends, as this is now being powered more and more through social media and, in particular, Instagram (Jaradat et al., 2018). Currently, 200 million users of Instagram follow at least one fashion account which $45 \%$ of them use to gain inspiration for fashion trends and present looks (Started, 2019).

Thence, fashion is an intricate field which, beyond garments and their imagery catalogues, brings together couture designers' visions, dressmakers' craft skills as well as mass-production short-cycle outputs, fashion media's trends, and individuals' aesthetic sensibilities. Although cloth prices still provide the most obvious constraint, the emphasis is actually placed upon consumers' ability to put together an interesting and individual outfit.

For this purpose, some individuals call upon fashion personal stylists to help them to look their best by curating clothing, makeup, and other aspects of personal style. To overcome economic or geographical 
Table 1: List of adopted clothing attributes and related labels.

\begin{tabular}{ll}
\hline Attribute & Labels \\
\hline Gender & Male, Female \\
Item & Bottom, Top, Dress \\
Occasion & Sport, Casual, Smart \\
Style & Blouse, Bodycon, Chinos, Hoodie, Jacket, Jeans, Joggies, Jumper, Leggings, Midi, PoloShirt, \\
& Shorts, Shirt, Skater, Skirt, Smock, Sweatshirt, Tie, Top, Trousers, T-shirt, Tux, Twill, Wrap \\
Fit & Muscle, Oversized, Petite, Skinny, Slim \\
Keyword & Bardot, Cable, Check, Chunky, Cold Shoulder, Crop, Drape, Floral, Funnel Neck, Leopard, \\
& Off Shoulder, Oversized, Padded, Pattern, Pencil, Ribbed, Stripe, Tapered, Teddy, Tie, Vneck \\
Brand & Adidas, Asos, Ax Paris, Barbor, Bershka, Boo Hoo, Boss, Daisy Street, Diesel, Farah, Fred Perry, \\
& French Connection, G-Star, Hollister, Homme, JDY, Lacoste, Lee Luke, Lee Malone, Lee Rider, \\
& Levis, Milk, Miss Guided, Miss Selfridge, Monki, Napapijri, New Look, Nike, Nudie, Only, \\
& Pier One, Pretty Little Thing, Puma, Ralph Lauren, Replay, River Island, Stradivarius, Ted Baker, \\
& Tommy, Vero, Vila, Warehouse \\
Colour & Black, Beige, Purple, Blue, Brown, Burgundy, Green, Grey, Nude, Orange, Pink, Red, Stone, \\
& White, Yellow \\
Sleeve & Sleeveless, Short, Long, 3/4 Length \\
Length & Short, Mini, Midi, Long \\
Material & Cotton, Denim, Leather, Polyamide, Polyester, Satin, Viscose, Wool \\
\hline
\end{tabular}

constraints, platforms such as Thread (Thread, 2019) can be used to bridge stylists and consumers, but they continue to mainly rely on human agents' services. Consequently, they can still be biased, e.g. by picking clothes the personal stylist likes and not necessary the ones the user likes. On the other hand, these services are not expandable at the rate that technologies based on Artificial Intelligence (AI) can (Luce, 2019).

Indeed, AI techniques can offer a scalable solution to a wide number of customers, while providing each of them with a personalized experience and bespoke advice (Luce, 2019). Hence, since the last decade (Chen et al., 2013), AI systems have started to be developed in the retail industry to find trends and patterns among data, especially for intelligent fashion analysis (Saponaro et al., 2018) such as clothing modeling (Kim and Cho, 1999), clothing recognition (Seo and Shin, 2018), clothing parsing (Yamaguchi et al., 2012), clothing retrieval (Rubio et al., 2017), clothing pairing (Stan and Mocanu, 2019), or clothing suggestion (Liu et al., 2014).

In particular, clothing recommendation, i.e. clothing pairing and/or clothing suggestion, can be performed by applying machine learning methods such as Neural Networks (NN) (Takagi et al., 2017), (Jaradat et al., 2018) or Support Vector Machines (SVM) (Chen et al., 2013), (Liu et al., 2014), (Quinn and Olszewska, 2019) as well as Self-Organizing Map (SOM) (Qian and Dong, 2010), (Hao and Hao, 2019) or association rules (Wakita et al., 2015). However, the resulting current fashion expert systems are mainly focused at making automated decisions on existing clothes within a customer's wardrobe, based on criteria such as a specified occasion (Liu et al., 2014), season (Hao and Hao, 2019), scenery (Jo et al., 2019), geo-location (Abe et al., 2018), garment colour (Tu and Dong, 2010) or brand (Wakita et al., 2015). Moreover, these recommenders rely on large amount of data that are not always available or could infringe on data privacy rights (Luce, 2019).

Actually, AI stylists face a number of challenges that makes fashion understanding complicated, such as its high level of variability (e.g. through time, seasons, climates, region, culture, etc.) (Takagi et al., 2017) and subjectivity (e.g. because of age, ethnicity, places visited, personal interests, mood, etc.) (Priyadharsun et al., 2018). A major task lies thus in the appropriate choice of attributes which describe the properties of the clothing (Zhao et al., 2017). Indeed, attributes are used both to learn a user's personal style and to make recommendations about what outfit this customer should wear. It is worth noting that attributes in fashion could be visual (Inoue et al., 2017), i.e. directly extracted from fashion images by means of computer-vision methods (Olszewska, 2019), or semantic ((Valle et al., 2018) with garments being often described by their materials, colors, patterns, fit, and cut (Al-Halah et al., 2017).

In this paper, we propose an AI-personal-stylist web application that embeds a Naive Bayesian Network (NBN) in order to determine suitable, new clothing suggestions in an automatic way, being given user's previous selections that are labeled with semantic features and taking into account customer's age and gender, which are provided by the user himself/herself at the registration stage of the application. 
The adopted machine learning method, i.e. NBN, is thus a supervised approach which requires only a small amount of data (voluntarily provided by the user) to train the system and which can intrinsically cope with missing data. Ultimately, our approach allows to enhance user's wardrobe by automatizing the personal-stylist recommendations of new outfits (i.e. emulating the AI stylist), while keeping the human in the loop (i.e. implicating the user), since 'The customer is the final filter. What survives the whole process is what people wear.' - Marc Jacobs.

Although the dominant field of fashion design is womenswear (Hsiao and Grauman, 2018), this work considers equally womenswear and menswear. On the other hand, the web application analyzed personal tastes of consumers (Liu and Shen, 2018) within both Western fashion catalogues and online images which were captured in the wild.

The resulting expert system provides a web-based solution, since internet has been proven to be important for fashion trends (Kitaura and Washida, 2015). Moreover, this intelligent fashion engine is an interactive tool, because human-computer interaction is a key to a successful recommender system (Wang et al., 2015) and because it is important to understand not only how algorithms see style but also how different individuals see style (Takagi et al., 2017).

Thus, the contributions of this paper are twofold. On one hand, as far as we know, we present the first study using Naive Bayesian Network for new clothing suggestion. On the other hand, we propose a novel web-based, interactive, AI-personal-stylist agent providing automated women/men fashion recommendations.

The paper is structured as follows. Section 2 describes the Naive Bayesian Network approach embedded in our web-based expert system for new clothing suggestion. Experiments are described in Section 3, while conclusions are drawn up in Section 4.

\section{PROPOSED AI-STYLIST RECOMMENDER}

The developed, AI-stylist expert system consists in an intelligent computer software that imitates human decision making, in this case the ones of a personal stylist, thanks to the use of a Naive Bayesian Network (NBN) as explained in Section 2.1. The resulting, automated recommender, that integrates this NBN, is presented in Section 2.2, with an emphasis on its design as well as its features such as web accessibility, user interactivity, and data security.

\subsection{Naive Bayesian Network}

The Naive Bayes (NB) model (Tan et al., 2018) is a probabilistic classifier that can perform estimations, while handle data uncertainty, i.e. the lack of exact knowledge due to inexact, incomplete, or even immeasurable data (Negnevitsky, 2011). Moreover, NB has the ability to process small datasets, i.e. c. $30+$ samples (Webb et al., 2005). These properties make the NB a suitable method for a fashion recommender, since fashion is highly variable and subjective. In particular, the use of NB theory in expert systems allows the computer to calculate independent assumptions. Actually, the NB model assumes that all attributes are conditionally independent given the class attribute. The fashion attributes as used in this work are presented in Table 1. Since each attribute is learned separately, it simplifies the teaching (or training phase) of the algorithm, which is based on the Bayes rule, as follows:

$$
P(H \mid E)=\frac{P(E \mid H) \cdot P(H)}{P(E)},
$$

where $P(H \mid E)$ is the posterior probability of hypothesis $H$ upon observing evidence $E$, i.e. the conditional probability that event $H$ occurs given that event $E$ has occurred; $P(E \mid H)$ is the likelihood, i.e. the probability that hypothesis $H$ being true will result in evidence $E ; P(H)$ is the class prior probability of hypothesis $H$ being true; and $P(E)$ is the predictor prior probability (Negnevitsky, 2011).

The Naive Bayesian Network (NBN) does not directly use results from the training phase, but applies parameters calculated from the training data to estimate the highest probability of the tested data in the expert system's testing phase. Thence, NBN can be used in the fashion recommender to calculate the probability of the user liking clothing suggestions based on his/her previous selections. Since the NB treats each attribute separately, this implies it copes well with missing data, e.g. in consequence of the user not having inputted all his/her characteristics or the user having provided only a partial (i.e. not exhaustive) list of his/her garment tastes, as it happens in real-world applications.

\subsection{Developed Expert System}

The present intelligent fashion recommender has been designed following the Dynamic Systems Development Method (DSDM) (Sommerville, 2015), which is an Agile approach consisting of four stages, namely, (i) business study and requirements, (ii) prototype iteration, (iii) design and development iteration, and (iv) implementation. 


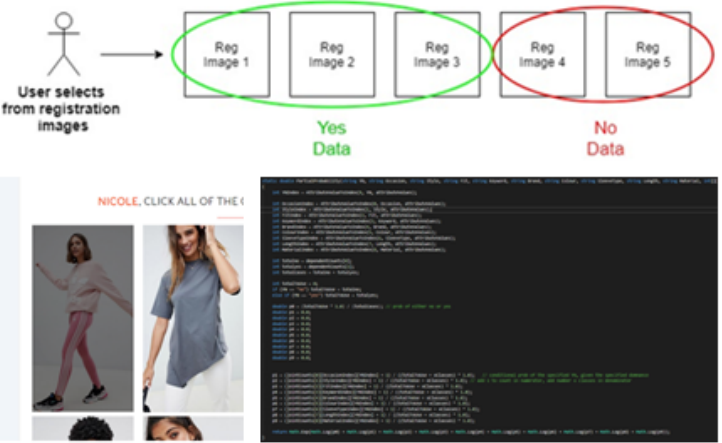

Figure 1: Overview of the training process of the Naive Bayesian Network.

In particular, the prototyping of the expert system involves the training and testing of the NBN algorithm which has been described in Section 2.1.

The NBN training, as depicted in Fig. 1, requires the user to select the clothing item(s) s/he likes among a database which is displayed within the application and which constitutes the training dataset. It is worth noting that the application is designed to ensure that the user picks at least one clothing item to feed the expert system with. Indeed, the NB algorithm needs yes/no data to be able to calculate a likelihood. Hence, the clothing selected by the user are the 'Yes' data, and the remaining clothing items in the training dataset that were not selected are the 'No' data.

In this application, the training dataset contains 113 records in total. Both genders have 40 registration images and a remaining 20 clothing items for females and 13 for males for the algorithm to select from. This results from the fact that women's clothing has tops, bottoms, and dresses in the app, whereas males only had tops and bottoms. On the other hand, each image of training data is labelled using the 11 attributes as presented in Table 1. There are other fields in the database such as cloth 'Price' which are kept in the database, but are not consider to be directly part of a user's fashion style model. The characteristics of each of the 11 attributes are collected from this database to be used in the frequency tables.

After a batch of new images, or testing datasest, is uploaded into the system, the NBN testing is performed as illustrated in Fig. 2. The NB algorithm is then run using the new added items and considering the previous yes/no data.

\section{EXPERIMENTS}

The developed AI-stylist agent integrating the proposed NBN approach into the web-based, interactive

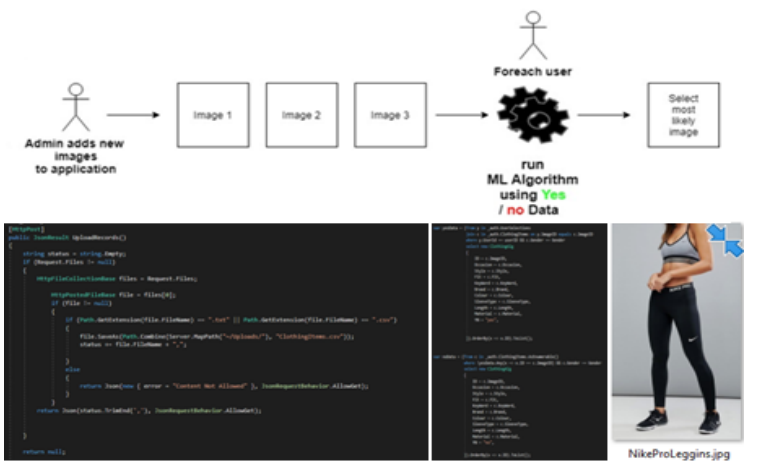

Figure 2: Overview of the testing process of the Naive Bayesian Network.

expert system has been evaluated both quantitatively and qualitatively in a series of experiments as described in Sections 3.1-3.3. It is worth noting that the NB algorithm has been extensively tested following the black box and white box approaches to ensure that the recommender was producing and outputting accurate results (as mentioned e.g. in the experiment 1 ), before it was released to the users for the usability testing (as reported in the experiment 2).

\subsection{Datasets and Equipment}

For all these experiments, two different datasets were used. Both of them comprise data reflecting diversity and equity, in order to minimize biases. Each dataset contains the 11 fields, namely, Gender, Item, Occasion, Style, Fit, Keyword, Brand, Colour, Sleeve, Length, and Material, as presented in Table 1. These fields are used in the algorithm for analysing patterns within the data to make prediction.

The first dataset is the primary database, which was used in the deployed application, and constitutes the main clothing catalogue that is based on Asos fashion e-commerce site, with Asos granting us the permission to use this data for the purpose of this research project. Hence, this dataset allowed us to apply our AI-stylist agent in real-world conditions.

The second dataset is composed of fashion images in the wild. This data was obtained from copyrightfree images which were retrieved from Google. This database is not only important to further assess the NBN performance, but it also allows to appraise our expert system integration with different sites or other applications in the future.

\subsection{Experiment 1}

The first series of experiments dwelt on the quantitative evaluation of the integration process of the entire 
system as schematized in Fig. 3. Some details of one of these tests are reported as below.

In this test, we assume at first that the NBN has been previously trained using sample-female customer's choices which were interactively provided during the registration phase. The resulting Clothing Catalogue Table is generated automatically and reported in Table 2 along with the used attributes which have been limited to 4 categories for this example. For each of these attributes, the likelihood is separately calculated and recorded in a table, as exemplified in Table 3 for the attribute Item.

Once the system is updated with new images of recently released fashion outfits as schematized in Fig. 3 , the NBN algorithm is processed to identify for a particular user what garment has the higher probability of 'Yes' within this data batch. Thence, the NBN algorithm is run for each of the image cloth in order to make a prediction on what the outcome of this user liking this specific clothing item (e.g. as described in Table 4) is. Considering the formerly computed likelihoods (e.g. Table 3) and based on the NB equation (Eq. 1), the posterior probability is computed for each evidence's value, as detailed below:

$$
\begin{aligned}
P(H \mid E=\text { Yes }) & \simeq P(\text { Item }=\text { Top } \mid H=\text { Yes }) \\
& \times P(\text { Occasion }=\text { Casual } \mid H=\text { Yes }) \\
& \times P(\text { Style }=\text { Jumper } \mid H=\text { Yes }) \\
& \times P(\text { Colour }=\text { Green } \mid H=\text { Yes }) \\
& \times P(\text { Hes }) \\
& =\frac{4}{10} \times \frac{9}{10} \times \frac{3}{10} \times \frac{1}{10} \times \frac{10}{20}, \\
P(H \mid E=N o) & \simeq P(\text { Item }=\text { Top } \mid H=\text { No }) \\
& \times P(\text { Occasion }=\text { Casual } \mid H=N o) \\
& \times P(\text { Style }=\text { Jumper } \mid H=N o) \\
& \times P(\text { Colour }=\text { Green } \mid H=N o) \\
& \times P(\text { H }=\text { No }) \\
& =\frac{6}{10} \times \frac{3}{10} \times \frac{0}{10} \times \frac{0}{10} \times \frac{10}{20},
\end{aligned}
$$

However, NB is not able to calculate any values= that have 0 , because it would in turn zero out the entire partial probability. This is known as the zerofrequency problem. A way to overcome it is to use the technique known as Laplacian Smoothing (or 1up smoothing) (Han et al., 2012); the previous results (from Eqs. 2-3) becoming as follows:

$$
\begin{aligned}
P(H \mid E=Y e s) & \simeq \frac{4}{10} \times \frac{9}{10} \times \frac{4}{11} \times \frac{2}{11} \times \frac{11}{22} \\
& \simeq 0.0119,
\end{aligned}
$$

Table 2: Example of Clothing Catalogue Table.

\begin{tabular}{llllc}
\hline Item & Occasion & Style & Colour & Decision \\
\hline Top & Casual & Tshirt & Grey & Yes \\
Bottom & Casual & Jeans & Blue & Yes \\
Bottom & Smart & Chinos & Black & Yes \\
Dress & Casual & Twill & Green & Yes \\
Dress & Casual & Jumper & Nude & Yes \\
Top & Casual & Jumper & Pink & Yes \\
Top & Casual & Jumper & Nude & Yes \\
Dress & Casual & Skater & Grey & Yes \\
Top & Casual & Tshirt & White & Yes \\
Dress & Casual & Bodycon & Black & Yes \\
Top & Casual & Tshirt & White & No \\
Bottom & Sport & Leggins & Black & No \\
Top & Smart & Tshirt & Red & No \\
Bottom & Sport & Leggins & Pink & No \\
Top & Sport & Tshirt & Pink & No \\
Dress & Smart & Wrap & Blue & No \\
Top & Sport & Tshirt & White & No \\
Dress & Casual & Wrap & Red & No \\
Top & Casual & Tshirt & Red & No \\
Top & Smart & Shirt & White & No \\
\hline
\end{tabular}

Table 3: Example of the Likelihood Table which has been generated based on Table 2 for the attribute Item.

\begin{tabular}{lcc}
\hline Item & \multicolumn{2}{c}{ Decision } \\
& Yes & No \\
\hline Top & $4 / 10$ & $6 / 10$ \\
Bottom & $2 / 10$ & $2 / 10$ \\
Dress & $4 / 10$ & $2 / 10$ \\
\hline
\end{tabular}

Table 4: Example of a new clothing item and its attribute values.

\begin{tabular}{llll}
\hline Item & Occasion & Style & Colour \\
\hline Top & Casual & Jumper & Green \\
\hline
\end{tabular}

$$
\begin{aligned}
P(H \mid E=N o) & \simeq \frac{6}{10} \times \frac{3}{10} \times \frac{1}{11} \times \frac{1}{11} \times \frac{11}{22} \\
& \simeq 0.0007 .
\end{aligned}
$$

Hence, the outcome of the NB prediction is the one with the highest posterior probability (in this case, the decision is a 'Yes', since $P(H \mid E=Y e s)>$ $P(H \mid E=N o))$. It is worth noting that Math.Log has been used when calculating the probabilities in order to prevent numeric errors that can occur with small real numerical values.

Lastly, the final output of the NBN processing, i.e. the item with the highest posterior probability of 'Yes' within the batch, is suggested to the user by sending her an email as displayed in Fig. 3. 


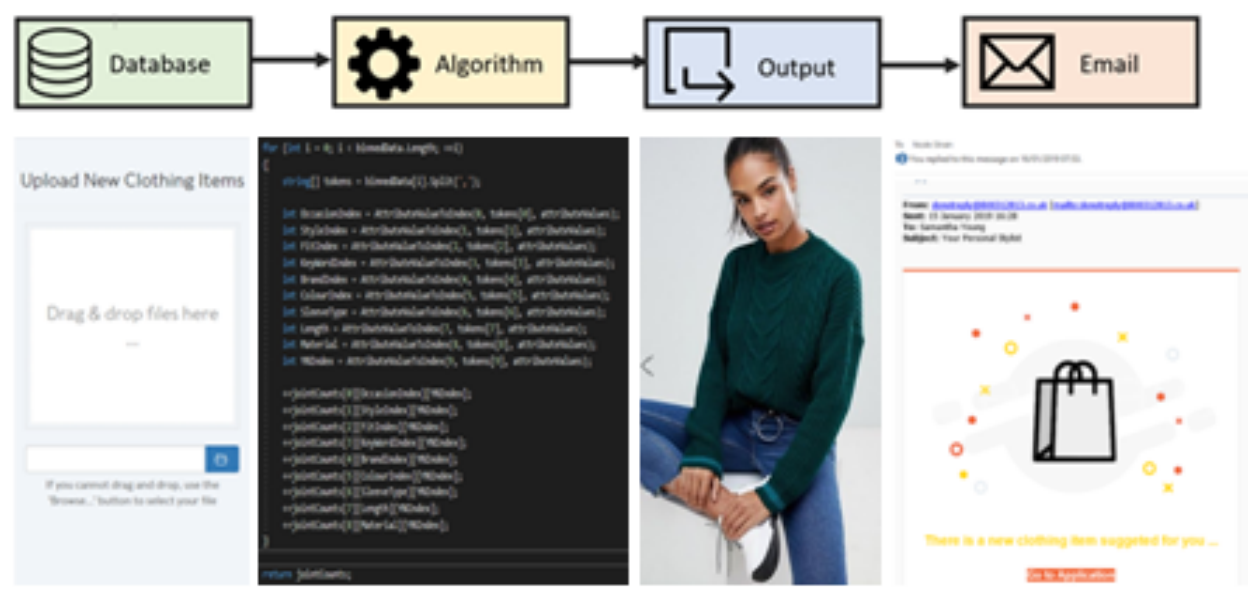

Figure 3: Example of integration test.

\subsection{Experiment 2}

The second type of experiments was focused on user testing. Indeed, the AI-agent must provide users with both an accurate algorithm for the clothe recommendations and a service that fashion customers would be interested to use.

For this purpose, a focus group, which was made of 12 volunteer people with mixed ages and different genders (i.e. 7 females and 5 males), has been contacted to perform the usability tests of our intelligent fashion application.

The group members were asked to interact with the recommender and, in particular at the registration phase, to select the images they liked from the clothing database in order to automatically build each user's style based on his/her choices. Then, they were asked to provide a comment about the suggested outfit which was emailed to them by the expert system as well as a feedback about their overall experience with the AI-stylist application.

The information gathered from the focus group at the registration phase was thus used as the training data for the supervised machine learning algorithm. After processing all of the focus group's images using the 'Image Name', 'User Gender', 'Cloth Price', and the fashion attributes (see Table 1), the decision was made automatically by the AI-agent for each of the user and sent by email to each of them, respectively. It is worth noting that, if the users were satisfied with the received clothing suggestion, the algorithm was considered as correct.

Thence, during the tests, each of these users was given instructions to take screenshots of the clothing items s/he selected (e.g. Figs. 4(a)-(b) and Figs. 4(d)(e), respectively) and of the clothing item that was suggested by the recommender (e.g. Fig. 4(c) and
Fig. 4(f), respectively). After the tests, all of the users were requested to complete a survey to assess if the application has passed the user testing and to rate their user's experience. The survey results have been anonymised and quantified as shown in Fig. 5. Overall, it appeared that the feedback about the clothing item recommended by the AI-agent was very positive (Fig. 5(a)). Moreover, all the users liked the usage of our intelligent fashion application (Fig. 5(b)).

\section{CONCLUSIONS}

In this paper, we proposed a new AI-stylist recommender which consists in an information filtering system that seeks to predict the 'rating', 'preference', or 'relevancy' that a user would give to an item not yet considered, using a model built on the characteristics of a provided set of this user's clothing items. Hence, our intelligent fashion expert system automatically suggests similar or related garments for a given customer by applying a Naive Bayesian Network (NBN). This supervised machine learning technique was trained based on user's inputs, while tested on different sets of fashion imagery as well as onlineretrieved images and deployed on data directly fed by current fashion e-catalogues. Hence, the NBN algorithm has been used for the first time in a clothingsuggestion, interactive web application, and the resulting, intelligent stylist agent has shown promising results in helping people to cope with fashion new trends and seasonal shifts. 


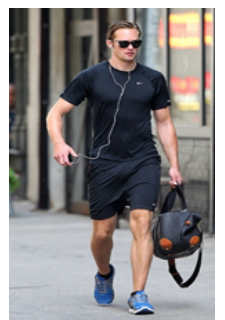

(a)

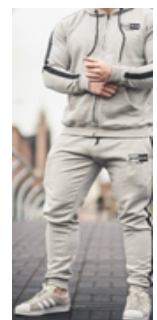

(b)

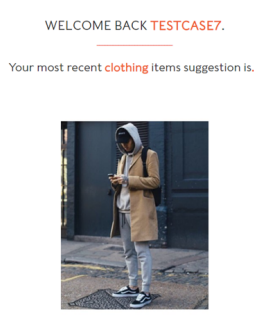

(c)

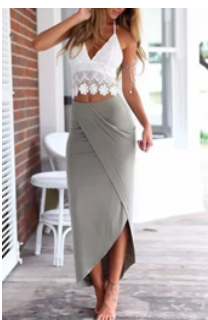

(d)

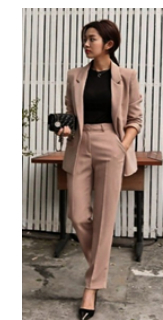

(e)

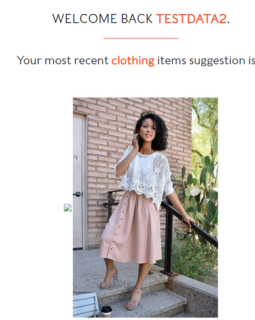

(f)

Figure 4: Examples of user testing for menswear and womenswear users, respectively. In particular, pictures (a)-(b) show a sample of a menswear user's given data within his application's account, and picture (c) is a sample of automatically suggested cloth data for the corresponding menswear user, while pictures (d)-(e) display a sample of a womenswear user's given data within her application's account, and picture (f) is a sample of automatically suggested cloth data for the corresponding womenswear user.

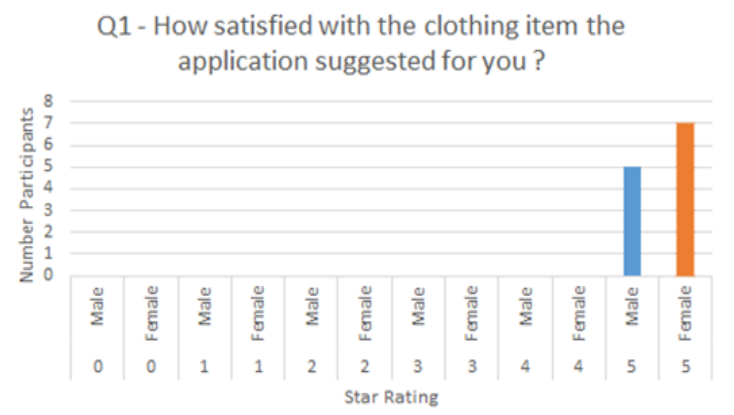

(a)
Q5 - Overall were you satisfied with the software?

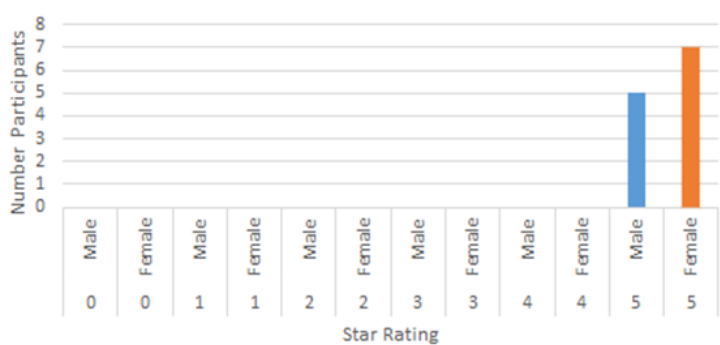

(b)

Figure 5: Excerpt of survey results.

\section{REFERENCES}

Abe, K., Minoguchi, M., Suzuki, T., Suzuki, T., Akimoto, N., Qiu, Y., Suzuki, R., Iwata, K., Satoh, Y., and Kataoka, H. (2018). Fashion culture database: Construction of database for world-wide fashion analysis. In IEEE International Conference on Control, Automation, Robotics, and Vision, pages 1721-1726.

Al-Halah, Z., Stiefelhagen, R., and Grauman, K. (2017). Fashion forward: Forecasting visual style in fashion. In IEEE International Conference on Computer Vision (ICCV), pages 388-397.

Arnold, R. (2009). Fashion: A Very Short Introduction. Oxford University Press.

Cecilio, D. (2015). E-commerce is changing the fashion industry: it's time to catch up. The Guardian. [online]. https://www.theguardian. com/small-business-network/2015/feb/06/.

Chen, Q., Wang, G., and Tan, C. L. (2013). Modeling fashion. In IEEE International Conference on Multimedia and Expo (ICME), pages 1-6.

Han, J., Kamber, M., and Rei, J. (2012). Data Mining: Concepts and Techniques. Morgan Kaufmann, 3rd edition.

Hao, L. and Hao, M. (2019). Design of intelligent clothing selection system based on neural network. In IEEE Information Technology, Networking, Electronic and Automation Control Conference, pages 1789-1792.
Hsiao, W.-L. and Grauman, K. (2018). Creating capsule wardrobes from fashion images. In IEEE International Conference on Computer Vision and Pattern Recognition (CVPR), pages 7161-7170.

Inoue, N., Simo-Serra, E., Yamasaki, T., and Ishikawa, H. (2017). Multi-label fashion image classification with minimal human supervision. In IEEE International Conference on Computer Vision Workshops (ICCV), pages 2261-2267.

Jaradat, S., Dokoohaki, N., Hammar, K., Wara, U., and Matskin, M. (2018). Dynamic CNN models for fashion recommendation in Instagram. In IEEE International Conference on Parallel and Distributed Processing with Applications, pages 1144-1151.

Jo, S.-Y., Jang, S.-H., Cho, H.-E., and Jeong, J.-W. (2019). Scenery-based fashion recommendation with crossdomain generative adversial networks. In IEEE International Conference on Big Data and Smart Computing, pages $1-4$.

Kim, H.-S. and Cho, S.-B. (1999). Development of an IGAbased fashion design aid system with domain specific knowledge. In IEEE International Conference on Systems, Man, and Cybernetics (SMC), pages III.663III.668.

Kitaura, S. and Washida, Y. (2015). Are geeks driving out the fashion industry? In IEEE International Conference on Management of Engineering and Technology, pages 1224-1229. 
Liu, S., Liu, L., and Yan, S. (2014). Fashion analysis: Current techniques and future directions. IEEE MultiMedia, 21(2):72-79.

Liu, Y. and Shen, Y. (2018). Personal tastes vs fashion trends: Predicting rating based on visual appearances and reviews. IEEE Access, 6:16655-16664.

Luce, L. (2019). Artificial Intelligence for Fashion: How AI is Revolutionizing the Fashion Industry. Apress.

Negnevitsky, M. (2011). Artificial Intelligence: A Guide to Intelligent Systems. Addison-Wesley, 3rd edition.

Olszewska, J. I. (2019). Designing transparent and autonomous intelligent vision systems. In International Conference on Agents and Artificial Intelligence (ICAART), pages 850-856.

Priyadharsun, S., Lakshigan, S., Baheerathan, S., Rajasooriyar, S., Rajapaksha, U., and Harshanath, S. (2018). Parade in the virtual dressing room. In IEEE International Conference on Computer Science and Education, pages 458-461.

Qian, S.-Q. and Dong, A.-H. (2010). Application of Kohonen self-organizing map in fashion parts classification. In WASE International Conference on Information Engineering, pages 226-229.

Quinn, M. and Olszewska, J. I. (2019). British sign language recognition in the wild based on multi-class SVM. In IEEE International Conference on Computer Science and Informatics, pages 81-86.

Rubio, A., Yu, L.-L., Simo-Serra, A., and Moreno-Noguer, F. (2017). Multi-modal joint embedding for fashion product retrieval. In IEEE International Conference on Image Processing (ICIP), pages 400-404.

Saponaro, M., Gal, D. L., Gao, M., Guisiano, M., and Maniere, I. C. (2018). Challenges and opportunities of artificial intelligence in the fashion world. In IEEE International Conference on Intelligent and Innovative Computing Applications, pages 1-5.

Seo, Y. and Shin, K.-S. (2018). Image classification of finegrained fashion image based on style using pre-trained convolutional neural network. In IEEE International Conference on Big Data Analysis, pages 387-390.

Sommerville, I. (2015). Software Engineering. Pearson, London, 10th edition.

Stan, C. and Mocanu, I. (2019). An intelligent personalized fashion recommendation system. In IEEE Interna- tional Conference on Control Systems and Computer Science, pages 210-215.

Started, G. (2019). The State of the Ecommerce Fashion Industry: Statistics, Trends and Strategy. [online]. https://www.shopify.com/enterprise/ ecommerce-fashion-industry.

Takagi, M., Simo-Serra, E., Iizuka, S., and Ishikawa, H. (2017). What makes a style: Experimental analysis of fashion prediction. In IEEE International Conference on Computer Vision Workshops (ICCV), pages 22472253.

Tan, P. N., Steinbach, M., and Kumar, V. (2018). Introduction to Data Mining. Pearson, 3rd edition.

Thread (2019). Stylist Recommendation Web Platform. [online]. https://www. thread.com.

Tu, Q. and Dong, L. (2010). An intelligent personalized fashion recommendation system. In IEEE International Conference on Communications, Circuits and Systems, pages 479-485.

Valle, D., Ziviani, N., and Veloso, A. (2018). Effective fashion retrieval based on semantic compositional networks. In IEEE International Joint Conference on Neural Networks (IJCNN), pages 1-8.

Wakita, Y., Oku, K., Huang, H.-H., and Kawagoe, K. (2015). A fashion-brand recommender system using brand association rules and features. In IEEE International Congress on Advanced Applied Informatics, pages 719-720.

Wang, L. C., Zeng, X. Y., Koehl, L., and Chen, Y. (2015). Intelligent fashion recommender system: Fuzzy logic in personalized garment design. IEEE Transactions on Human-Machine Systems, 45(1):95-109.

Webb, G., Boughton, J., and Wang, Z. (2005). Not so Naive Bayes: Aggregating one-dependence estimators. $\mathrm{Ma}$ chine Learning, 58(1):5-24.

Yamaguchi, K., Kiapour, H. H., Ortiz, L. E., and Berg, T. L. (2012). Parsing clothing in fashion photographs. In IEEE International Conference on Computer Vision and Pattern Recognition (CVPR), pages 3570-3577.

Zhao, B., Feng, J., Wu, X., and Yan, S. (2017). Memoryaugmented attribute manipulation networks for interactive fashion search. In IEEE International Conference on Computer Vision and Pattern Recognition (CVPR), pages 6156-6164. 